\title{
Development of honey hydrogel dressing for enhanced wound healing
}

\begin{abstract}
Radiation at 25 and $50 \mathrm{kGy}$ showed no effect on the acidic $\mathrm{pH}$ of the local honey, Gelam, and its antimicrobial property against Staphylococcus aureus but significantly reduced the viscosity. Honey stored up to 2 years at room temperature retained all the properties studied. Radiation sterilized Gelam honey significantly stimulated the rate of burn wound healing in Sprague-Dawley rats as demonstrated by the increased rate of wound contraction and gross appearance. Gelam honey attenuates wound inflammation; and re-epithelialization was well advanced compared to the treatment using silver sulphadiazine (SSD) cream. To enhance further the use of honey in wound treatment and for easy handling, Gelam honey was incorporated into our hydrogel dressing formulation, which was then cross-linked and sterilized using electron beam at $25 \mathrm{kGy}$. Hydrogel with $6 \%$ of honey was selected based on the physical appearance.
\end{abstract}

Keyword: Honey, Radiation sterilization, Cross-linking, Hydrogel 